\title{
A Versatile Approach Toward the Ansamycin Antibiotics
}

\author{
Weimin Peng and Brian S. J. Blagg* \\ Department of Medicinal Chemistry and The Center for Chemical Methodology and \\ Library Development, The University of Kansas, 1251 Wescoe Hall Drive, Malott 4070, \\ Lawrence, Kansas 66045-7563 \\ bblagg@ku.edu
}

Supplementary information for the preparation of trienomycin A analogues 
<smiles>O=[N+]([O-])c1cc(CO)cc(OCc2ccccc2)c1</smiles>

[3-(benzyloxy)-5-nitrophenyl]methanol. To a suspension of benzyl alcohol 1 (1.47 g, $8.69 \mathrm{mmol})$ and $\mathrm{K}_{2} \mathrm{CO}_{3}(1.96 \mathrm{~g} \mathrm{~g}, 14.16 \mathrm{mmol})$ in acetone $(80 \mathrm{~mL})$ was added benzyl bromide (1.05 g, $1.08 \mathrm{~mL}, 9.12 \mathrm{mmol})$. The mixture was heated at reflux for $15 \mathrm{~h}$, concentrated, and water $(80 \mathrm{~mL})$ added. The mixture was extracted with EtOAc $(3 \times 50$ $\mathrm{mL})$ and the combined organic layers washed with saturated aqueous $\mathrm{NaCl}(80 \mathrm{~mL})$, dried $\left(\mathrm{Na}_{2} \mathrm{SO}_{4}\right)$, filtered, and concentrated. The residue was purified via chromatography $\left(\mathrm{SiO}_{2}, 50 \% \mathrm{EtOAc}\right.$ in hexanes) to afford [3-(benzyloxy)-5-nitrophenyl]methanol $(2.05 \mathrm{~g}$, $89 \%$ yield) as a pale yellow oil: ${ }^{1} \mathrm{H} \mathrm{NMR}\left(\mathrm{CDCl}_{3}, 400 \mathrm{MHz}\right) \delta 7.82(\mathrm{~s}, 1 \mathrm{H}), 7.71(\mathrm{~s}, 1 \mathrm{H})$, $7.41(\mathrm{~m}, 5 \mathrm{H}), 7.26(\mathrm{~s}, 1 \mathrm{H}), 5.13(\mathrm{~s}, 2 \mathrm{H}), 4.76(\mathrm{~s}, 2 \mathrm{H}), 1.98(\mathrm{~s}, 1 \mathrm{H},-\mathrm{OH}) ;{ }^{13} \mathrm{C} \mathrm{NMR}$ $\left(\mathrm{CDCl}_{3}, 125 \mathrm{MHz}\right) \delta 158.3,148.3,142.9,134.5,127.8(2 \mathrm{C}), 127.4,126.6(2 \mathrm{C}), 118.6$, 113.0, 107.2, 69.7, 63.0; IR (film) $v_{\max } 3328,3109,2867,1631,1579,1529,1496,1465$, $1452,1394,1348,1330,1280,1238,1153,1099,1026,952,935,862,844,783,746,696$ $\mathrm{cm}^{-1}$; HRMS (TOF-FAB+) found 266.1013 $\left(\mathrm{M}+\mathrm{Li}^{+}\right)$, calcd 266.1005 for $\mathrm{C}_{14} \mathrm{H}_{13} \mathrm{NO}_{4} \mathrm{Li}$.<smiles>O=Cc1cc(OCc2ccccc2)cc([N+](=O)[O-])c1</smiles>

3-(benzyloxy)-5-nitrobenzaldehyde (2). $4 \AA$ Molecular sieves $(2.5 \mathrm{~g})$ were added to a solution of [3-(benzyloxy)-5-nitrophenyl]methanol $(2.05 \mathrm{~g}, \quad 7.90 \mathrm{mmol})$ in dichloromethane $(120 \mathrm{~mL})$ at $0{ }^{\circ} \mathrm{C}$. The mixture was stirred for $5 \mathrm{~min}$ before pyridinium chlorochromate $(2.55 \mathrm{~g}, 11.85 \mathrm{mmol})$ was added over $5 \mathrm{~min}$. The mixture was stirred at $\mathrm{rt}$ 
for $4 \mathrm{~h}$ before it was diluted with diethyl ether $(120 \mathrm{~mL})$ and filtered over $\mathrm{SiO}_{2}$. The green solution was concentrated, and the residue purified via chromatography $\left(\mathrm{SiO}_{2}, 20 \%\right.$ EtOAc in hexanes) to afford 2 (2.01 g, $99 \%$ yield) as a pale yellow solid: ${ }^{1} \mathrm{H}$ NMR $\left(\mathrm{CDCl}_{3}, 400 \mathrm{MHz}\right) \delta 10.1(\mathrm{~s}, 1 \mathrm{H}), 8.31(\mathrm{~s}, 1 \mathrm{H}), 8.07(\mathrm{~s}, 1 \mathrm{H}), 7.79(\mathrm{~s}, 1 \mathrm{H}), 7.43(\mathrm{~m}, 5 \mathrm{H})$,

$5.16(\mathrm{~s}, 2 \mathrm{H}) ;{ }^{13} \mathrm{C} \mathrm{NMR}\left(\mathrm{CDCl}_{3}, 125 \mathrm{MHz}\right) \delta 189.6,159.9,149.7,138.2,135.0,128.9$ (2C), 128.7, 127.7 (2C), 120.1, 117.3, 115.4, 71.2; IR (film) $v_{\max } 3097,2869,1701,1618$, $1585,1533,1496,1456,1446,1392,1353,1280,1245,1149,1091,1080,1054$, $1027,923,881,784,738,696,657,466 \mathrm{~cm}^{-1}$;<smiles>CCOC(=O)C=Cc1cc(OCC)cc([N+](=O)[O-])c1</smiles>

(E)-ethyl 3-[3-(benzyloxy)-5-nitrophenyl]acrylate (4). To a suspension of 60\% $\mathrm{NaH}$ (396 $\mathrm{mg}, 9.89 \mathrm{mmol})$ in anhydrous THF $(40 \mathrm{~mL})$ at $\mathrm{rt}$ was added triethyl phosphonoacetate $(2.03 \mathrm{~g}, 1.81 \mathrm{~mL}, 9.07 \mathrm{mmol})$. The mixture was stirred for $30 \mathrm{~min}$ before aldehyde $2(2.12 \mathrm{~g}, 8.24 \mathrm{mmol})$ was added over $5 \mathrm{~min}$ at $0{ }^{\circ} \mathrm{C}$. The mixture was stirred at $\mathrm{rt}$ for $3 \mathrm{~h}$ before addition of saturated aqueous $\mathrm{NH}_{4} \mathrm{Cl}$ solution $(40 \mathrm{~mL})$. The organic solvent was removed, and the mixture extracted with EtOAc $(3 \times 50 \mathrm{~mL})$. The combined organic layers were washed with saturated aqueous $\mathrm{NaCl}(80 \mathrm{~mL})$, dried $\left(\mathrm{Na}_{2} \mathrm{SO}_{4}\right)$, filtered, and concentrated. The residue was purified via recrystallization with EtOAc and hexanes to afford 4 (2.56 g, $95 \%$ yield) as a white solid: ${ }^{1} \mathrm{H} \mathrm{NMR}\left(\mathrm{CDCl}_{3}\right.$, $400 \mathrm{MHz}) \delta 8.00(\mathrm{~s}, 1 \mathrm{H}), 7.81(\mathrm{~s}, 1 \mathrm{H}), 7.64(\mathrm{~d}, J=16.0 \mathrm{~Hz}, 1 \mathrm{H}), 7.40(\mathrm{~m}, 6 \mathrm{H}), 6.52(\mathrm{~d}, J$ $=16.0 \mathrm{~Hz}, 1 \mathrm{H}), 5.17(\mathrm{~s}, 2 \mathrm{H}), 4.28(\mathrm{q}, J=7.1 \mathrm{~Hz}, 2 \mathrm{H}), 1.35(\mathrm{t}, J=7.1 \mathrm{~Hz}, 3 \mathrm{H}) ;{ }^{13} \mathrm{C} \mathrm{NMR}$ $\left(\mathrm{CDCl}_{3}, 125 \mathrm{MHz}\right) \delta 166.1,159.5,149.6,141.8,137.0,135.4,128.9$ (2C), 128.5, 127,7 
(2C), 121.6, 120.9, 115.0, 110.3, 70.8, 61.0, 14.3; IR (film) $v_{\max } 3407,2981,1722,1645$, $1573,1525,1444,1390,1357,1317,1272,1240,1182,1029,748 \mathrm{~cm}^{-1}$; HRMS (TOF$\mathrm{FAB}+)$ found $328.1164\left(\mathrm{M}+\mathrm{H}^{+}\right)$, calcd 328.1185 for $\mathrm{C}_{18} \mathrm{H}_{17} \mathrm{NO}_{5}$.

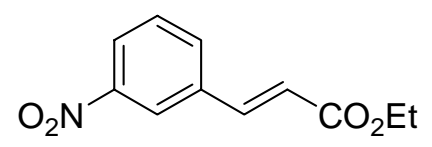

(E)-ethyl 3-(3-nitrophenyl)acrylate (5) ${ }^{\mathrm{a}} \cdot{ }^{1} \mathrm{H} \mathrm{NMR}\left(\mathrm{CDCl}_{3}, 400 \mathrm{MHz}\right) \delta 8.40(\mathrm{~s}, 1 \mathrm{H})$, $8.25(\mathrm{~d}, J=8.2 \mathrm{~Hz}, 1 \mathrm{H}), 7.84(\mathrm{~d}, J=7.7 \mathrm{~Hz}, 1 \mathrm{H}), 7.74(\mathrm{~d}, J=16.0 \mathrm{~Hz}, 1 \mathrm{H}), 7.61(\mathrm{dd}, J$ $=8.2,7.7 \mathrm{~Hz}, 1 \mathrm{H}), 6.58(\mathrm{~d}, J=16.0 \mathrm{~Hz}, 1 \mathrm{H}), 4.31(\mathrm{q}, J=7.1 \mathrm{~Hz}, 2 \mathrm{H}), 1.37(\mathrm{t}, J=7.1 \mathrm{~Hz}$, $3 \mathrm{H})$.<smiles>CCOCCc1cc(OCC)cc([N+](=O)[O-])c1</smiles>

ethyl 3-[3-(benzyloxy)-5-nitrophenyl]propanoate (6). A solution of acetic acid (0.43 g, $0.41 \mathrm{~mL}, 7.18 \mathrm{mmol})$ in DME $(35 \mathrm{~mL})$ was added to a suspension of potassium diazodicarboxylate $(1.39 \mathrm{~g}, 7.18 \mathrm{mmol})$ and $4(0.78 \mathrm{~g}, 2.39 \mathrm{mmol})$ in DME $(20 \mathrm{~mL})$ dropwise at $50{ }^{\circ} \mathrm{C}$. The mixture was stirred for $48 \mathrm{~h}$ at $50{ }^{\circ} \mathrm{C}$ before it was cooled to rt and filtered. The eluent was concentrated and the residue purified via chromatography $\left(\mathrm{SiO}_{2}, 25 \%\right.$ EtOAc in hexanes) to afford $6(0.76 \mathrm{~g}, 97 \%$ yield $)$ as a pale yellow oil: ${ }^{1} \mathrm{H}$ NMR $\left(\mathrm{CDCl}_{3}, 400 \mathrm{MHz}\right) \delta 7.70(\mathrm{~m}, 1 \mathrm{H}), 7.66(\mathrm{~m}, 1 \mathrm{H}), 7.41(\mathrm{~m}, 5 \mathrm{H}), 7.16(\mathrm{~m}, 1 \mathrm{H}), 5.12$ (s, 2H), $4.13(\mathrm{q}, J=7.1 \mathrm{~Hz}, 2 \mathrm{H}), 3.01(\mathrm{t}, J=7.5 \mathrm{~Hz}, 2 \mathrm{H}), 2.65(\mathrm{q}, J=7.5 \mathrm{~Hz}, 2 \mathrm{H}), 1.24$ $(\mathrm{t}, J=7.1 \mathrm{~Hz}, 3 \mathrm{H}) ;{ }^{13} \mathrm{C} \mathrm{NMR}\left(\mathrm{CDCl}_{3}, 125 \mathrm{MHz}\right) \delta 171.2,158.2,148.2,142.5,134.7$, 128.0 (2C), 127.5, 126.8 (2C), 121.1, 115.2, 106.1, 69.6, 59.7, 34.0, 29.6, 13.2; IR (film) 
$v_{\max } 3440,3103,2979,1728,1629,1577,1523,1352,1303,1271,1240,1186,1157$, 1058, 1024, 869, 860, 746, 730, $694 \mathrm{~cm}^{-1}$; HRMS (TOF-FAB+) found 330.1324 $\left(\mathrm{M}+\mathrm{H}^{+}\right)$, calcd 330.1341 for $\mathrm{C}_{18} \mathrm{H}_{20} \mathrm{NO}_{5}$.

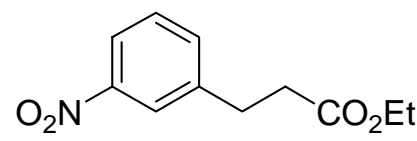

ethyl 3-(3-nitrophenyl)propanoate $(7)^{\mathrm{b}} \cdot{ }^{1} \mathrm{H} \mathrm{NMR}\left(\mathrm{CDCl}_{3}, 400 \mathrm{MHz}\right) \delta 8.11(\mathrm{~m}, 2 \mathrm{H})$, $7.57(\mathrm{~d}, J=7.9 \mathrm{~Hz}, 1 \mathrm{H}), 7.48(\mathrm{~m}, 1 \mathrm{H}), 4.15(\mathrm{q}, J=7.1 \mathrm{~Hz}, 2 \mathrm{H}), 3.08(\mathrm{t}, J=7.5 \mathrm{~Hz}, 2 \mathrm{H})$, $2.70(\mathrm{t}, J=7.5 \mathrm{~Hz}, 2 \mathrm{H}), 1.26(\mathrm{t}, J=7.1 \mathrm{~Hz}, 3 \mathrm{H})$.<smiles>O=[N+]([O-])c1cc(CCCO)cc(Cc2ccccc2)c1</smiles>

3-[3-(benzyloxy)-5-nitrophenyl]propan-1-ol (8). Diisobutylaluminum hydride (5.0 mL, $5 \mathrm{mmol}, 1 \mathrm{M}$ in hexane) was slowly added to a solution of ester $6(0.83 \mathrm{~g}, 2.51 \mathrm{mmol})$ in toluene $(45 \mathrm{~mL})$ at $-78{ }^{\circ} \mathrm{C}$. The resulting mixture was warmed to $0{ }^{\circ} \mathrm{C}$ and stirred for $4 \mathrm{~h}$ before quenching with a saturated solution of potassium sodium tartrate $(40 \mathrm{~mL})$. The heterogeneous mixture was stirred for $2 \mathrm{~h}$ and then extracted with ethyl acetate $(3 \times 40$ $\mathrm{mL})$. The combined organic layers were washed with saturated aqueous $\mathrm{NaCl}(70 \mathrm{~mL})$, dried $\left(\mathrm{Na}_{2} \mathrm{SO}_{4}\right)$, filtered, and concentrated. The residue was purified via chromatography $\left(\mathrm{SiO}_{2}, 50 \%\right.$ EtOAc in hexanes $)$ to afford $\mathbf{8}(0.51 \mathrm{~g}, 71 \%$ yield $)$ as a pale yellow oil: ${ }^{1} \mathrm{H}$ NMR $\left(\mathrm{CDCl}_{3}, 400 \mathrm{MHz}\right) \delta 7.70(\mathrm{~s}, 1 \mathrm{H}), 7.66(\mathrm{~m}, 1 \mathrm{H}), 7.41(\mathrm{~m}, 5 \mathrm{H}), 7.15(\mathrm{~s}, 1 \mathrm{H}), 5.12(\mathrm{~s}$, 2H), $3.67(\mathrm{t}, J=6.3 \mathrm{~Hz}, 2 \mathrm{H}), 2.79(\mathrm{t}, J=7.7 \mathrm{~Hz}, 2 \mathrm{H}), 1.90(\mathrm{tt}, J=7.7,6.3 \mathrm{~Hz}, 2 \mathrm{H}), 1.46$ $(\mathrm{s}, 1 \mathrm{H},-\mathrm{OH}) ;{ }^{13} \mathrm{C} \mathrm{NMR}\left(\mathrm{CDCl}_{3}, 100 \mathrm{MHz}\right) \delta 159.5,149.6,145.2,136.2,129.2(2 \mathrm{C})$, 
128.8, 128.0 (2C), 122.6, 116.6, 107.1, 71.0, 62.1, 33.9, 32.2; IR (film) $v_{\max } 3367,2937$, $2869,1623,1577,1525,1454,1348,1274,1155,1027,908,862,783,746,696 \mathrm{~cm}^{-1}$

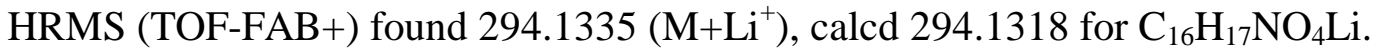<smiles>O=[N+]([O-])c1cccc(CCCO)c1</smiles>

3-(3-nitrophenyl)propan-1-ol (9) ${ }^{\mathrm{c}} .{ }^{1} \mathrm{H}$ NMR $\left(\mathrm{CDCl}_{3}, 400 \mathrm{MHz}\right) \delta 8.09(\mathrm{~m}, 2 \mathrm{H}), 7.56(\mathrm{~d}$, $J=7.6 \mathrm{~Hz}, 1 \mathrm{H}), 7.47(\mathrm{~m}, 1 \mathrm{H}), 3.71(\mathrm{t}, J=6.3 \mathrm{~Hz}, 2 \mathrm{H}), 2.86(\mathrm{t}, J=7.8 \mathrm{~Hz}, 2 \mathrm{H}), 1.95(\mathrm{tt}, J$ $=7.8,5.3 \mathrm{~Hz}, 2 \mathrm{H}), 1.62(\mathrm{~s}, 1 \mathrm{H},-\mathrm{OH})$.<smiles>O=[N+]([O-])c1cc(CCCI)cc(OCc2ccccc2)c1</smiles>

1-benzyloxy-3-(3-iodopropyl)-5-nitrobenzene (10). To a solution of 8 (1.03 g, 3.59 mmol) in THF (21 mL) and $\mathrm{CH}_{3} \mathrm{CN}(84 \mathrm{~mL})$ was added triphenyl phosphine (1.88 g, 7.18 mmol) and imidazole $(0.98 \mathrm{~g}, 14.37 \mathrm{mmol})$ at $0{ }^{\circ} \mathrm{C}$. The mixture was stirred for $10 \mathrm{~min}$ before iodine ( $3.65 \mathrm{~g}, 14.37 \mathrm{mmol})$ was added. The resulting solution was warmed to rt and stirred for $3 \mathrm{~h}$ before quenching with an aqueous solution of sodium thiosulfate (20 $\mathrm{mL}$ ). The organic solvents were removed and the aqueous solution extracted with diethyl ether $(3 \times 50 \mathrm{~mL})$. The combined organic layers were washed with saturated aqueous $\mathrm{NaCl}(80 \mathrm{~mL})$, dried $\left(\mathrm{Na}_{2} \mathrm{SO}_{4}\right)$, filtered, and concentrated. The residue was purified via chromatography $\left(\mathrm{SiO}_{2}, 10 \%\right.$ EtOAc in hexanes) to afford $\mathbf{1 0}(1.32 \mathrm{~g}, 93 \%$ yield $)$ as a yellow oil: ${ }^{1} \mathrm{H}$ NMR $\left(\mathrm{CDCl}_{3}, 400 \mathrm{MHz}\right) \delta 7.69(\mathrm{~m}, 2 \mathrm{H}), 7.42(\mathrm{~m}, 5 \mathrm{H}), 7.15(\mathrm{~s}, 1 \mathrm{H}), 5.13$ (s, 2H), 3.16 (t, $J=6.7 \mathrm{~Hz}, 2 \mathrm{H}), 2.80(\mathrm{t}, J=7.4 \mathrm{~Hz}, 2 \mathrm{H}), 2.12(\mathrm{tt}, J=7.4,6.7 \mathrm{~Hz}, 2 \mathrm{H})$; 
${ }^{13} \mathrm{C} \mathrm{NMR}\left(\mathrm{CDCl}_{3}, 100 \mathrm{MHz}\right) \delta 159.6,149.7,143.8,136.1,129.2(2 \mathrm{C}), 128.9,128.1(2 \mathrm{C})$, 122.7, 116.6, 107.4, 71.0, 36.4, 34.5, 5.8; IR (film) $v_{\max } 3089,2933,2866,1625,1579$, 1529, 1452, 1350, 1272, 1213, 1155, 1041, 869, 781, 746, 698, $682 \mathrm{~cm}^{-1}$; HRMS (TOF$\mathrm{FAB}+$ ) found $398.0238\left(\mathrm{M}+\mathrm{H}^{+}\right)$, calcd 398.0253 for $\mathrm{C}_{16} \mathrm{H}_{17} \mathrm{INO}_{3}$.<smiles>O=[N+]([O-])c1cccc(CCCI)c1</smiles>

1-(3-iodopropyl)-3-nitrobenzene (11). 11 was prepared from 9 in analogous fashion as described for iodide $\mathbf{1 0}$ to give $\mathbf{1 1}$ in $84 \%$ yield as a pale yellow oil: ${ }^{1} \mathrm{H} \mathrm{NMR}\left(\mathrm{CDCl}_{3}\right.$, $400 \mathrm{MHz}) \delta 8.08(\mathrm{~m}, 2 \mathrm{H}), 7.55(\mathrm{~m}, 1 \mathrm{H}), 7.47(\mathrm{~m}, 1 \mathrm{H}), 3.18(\mathrm{t}, J=6.7 \mathrm{~Hz}, 2 \mathrm{H}), 2.86(\mathrm{t}, J$ $=7.5 \mathrm{~Hz}, 2 \mathrm{H}), 2.16(\mathrm{tt}, J=6.7,7.5 \mathrm{~Hz}, 2 \mathrm{H}) ;{ }^{13} \mathrm{C} \mathrm{NMR}\left(\mathrm{CDCl}_{3}, 100 \mathrm{MHz}\right) \delta 148.8,142.8$, 135.3, 129.9, 123.8, 121.9, 36.3, 34.7, 5.8; IR (film) $v_{\max } 2923,2854,1525,1448,1350$, 1213, $732 \mathrm{~cm}^{-1}$; HRMS (TOF-FAB+) found $291.9822\left(\mathrm{M}+\mathrm{H}^{+}\right)$, calcd 291.9834 for $\mathrm{C}_{9} \mathrm{H}_{10} \mathrm{INO}_{2}$.

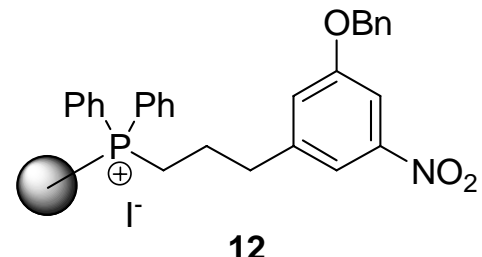

12

Resin-bound phosphonium salt 12. Resin bound triphenylphosphine (1.05 g, 1.4 2.0 $\mathrm{mmol}, 1.4 \sim 2.0 \mathrm{mmol} / \mathrm{g})$ was added to a solution of $10(0.69 \mathrm{~g}, 1.73 \mathrm{mmol})$ in degassed DMF (10 mL). The mixture was heated at $70{ }^{\circ} \mathrm{C}$ and shaken for 24 hours. The resin was filtered and washed successively with DMF $(20 \mathrm{~mL}), \mathrm{CH}_{2} \mathrm{Cl}_{2}(20 \mathrm{~mL})$ and diethyl ether $(20 \mathrm{~mL})$ five times. The resin was dried at $100{ }^{\circ} \mathrm{C}$ for $24 \mathrm{~h}$ to afford $\mathbf{1 2}(1.30 \mathrm{~g})$. 


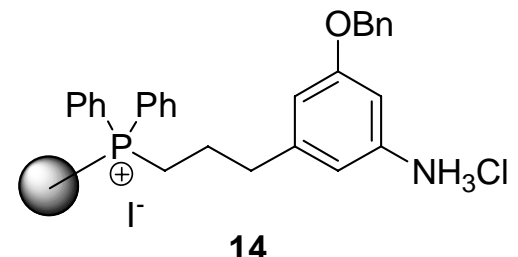

Reduction of nitrated resin 12 with $\mathrm{SnCl}_{2}$ to afford 14. A solution of $\mathrm{SnCl}_{2}(0.79 \mathrm{~g}$, $4.14 \mathrm{mmol})$ in DMF (5 mL) was added to a suspension of resin $\mathbf{1 2}(1.30,0.62 \mathrm{mmol})$ in DMF $(10 \mathrm{~mL})$, followed by the addition of water $(0.15 \mathrm{~mL}, 8.28 \mathrm{mmol})$. The mixture was shaken for $12 \mathrm{~h}$ at $\mathrm{rt}$ before the solvent was decanted. The above procedure was repeated two additional times. After which, the resin was filtered and washed successively with DMF (20 mL), $\mathrm{CH}_{2} \mathrm{Cl}_{2}(20 \mathrm{~mL})$ and ether $(20 \mathrm{~mL})$ five times and then dried at $100{ }^{\circ} \mathrm{C}$ for 24 hours to afford the aniline resin $\mathbf{1 4}$.

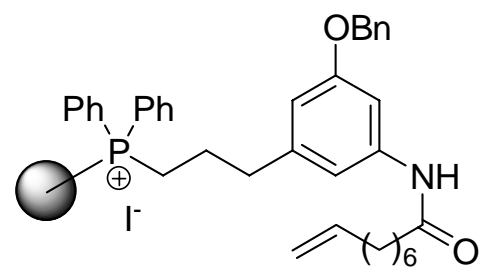

18

Coupling of acid chloride with resin bound aniline. Dimethylaminopyridine ( $0.30 \mathrm{~g}$, $2.48 \mathrm{mmol})$ was added to a suspension of resin $14(0.62 \mathrm{mmol})$ in anhydrous DMF (10 $\mathrm{mL}$ ). Once the DMAP had completely dissolved, 8-nonenoyl chloride (0.87 g, $4.97 \mathrm{mmol})$ was added and the mixture was shaken vigorously for $2 \mathrm{~d}$ at $\mathrm{rt}$. The resin was filtered and washed successively with DMF (20 mL), $\mathrm{CH}_{2} \mathrm{Cl}_{2}(20 \mathrm{~mL})$ and ether $(20 \mathrm{~mL})$ five times and then dried at $100{ }^{\circ} \mathrm{C}$ for $24 \mathrm{~h}$ to afford the resin-bound amide 18 . 
Following the same three-step procedure and starting with iodide 11, the resin-bound amides 16 and 17 were prepared.<smiles>C=C[Y10](=O)C(=O)Nc1cc(CCC=C(C)C)cc(OCc2ccccc2)c1</smiles>

Typical procedure for intermolecular Wittig reaction. Sodium bis(trimethylsilyl)amide (101 uL, $0.20 \mathrm{mmol}, 2 \mathrm{M}$ in THF) was added dropwise to a suspension of resinbound amide 18 (202 mg, $0.09 \mathrm{mmol})$ and 7-octen-2-one (11.6 mg, $14 \mathrm{uL}, 0.09 \mathrm{mmol})$ in $\operatorname{DMF}(0.9 \mathrm{~mL})$ at $0{ }^{\circ} \mathrm{C}$. The mixture was warmed to $\mathrm{rt}$ and stirred for $4 \mathrm{~h}$. The resin was filtered and washed successively with $\mathrm{DMF}(2 \mathrm{~mL} \times 3)$, saturated aqueous $\mathrm{NH}_{4} \mathrm{Cl}$ solution $(2 \mathrm{~mL} \times 3)$, and with $\mathrm{DMF}(2 \mathrm{~mL} \times 3)$. The filtrate was extracted with diethyl ether $(3 \times 20 \mathrm{~mL})$ and the combined organic layers were washed with saturated aqueous $\mathrm{NaCl}(40 \mathrm{~mL})$, dried $\left(\mathrm{Na}_{2} \mathrm{SO}_{4}\right)$, filtered, and concentrated. The residue was purified via chromatography $\left(\mathrm{SiO}_{2}, 20 \%\right.$ EtOAc in hexanes) to afford $26(12.4 \mathrm{mg})$ as a mixture of isomers and a yellow oil: ${ }^{1} \mathrm{H}$ NMR $\left(\mathrm{CDCl}_{3}, 400 \mathrm{MHz}\right) \delta 7.40(\mathrm{~m}, 5 \mathrm{H}), 7.27(\mathrm{~m}, 1 \mathrm{H}), 7.08$ $(\mathrm{s}, 1 \mathrm{H}), 6.81(\mathrm{~m}, 1 \mathrm{H}), 6.59(\mathrm{~s}, 1 \mathrm{H}), 5.81(\mathrm{~m}, 2 \mathrm{H}), 5.14(\mathrm{t}, J=6.9 \mathrm{~Hz}, 1 \mathrm{H}), 5.04(\mathrm{~s}, 2 \mathrm{H})$, $4.97(\mathrm{~m}, 4 \mathrm{H}), 2.55(\mathrm{~m}, 2 \mathrm{H}), 2.31(\mathrm{~m}, 4 \mathrm{H}), 2.02(\mathrm{~m}, 6 \mathrm{H}), 1.71(\mathrm{~m}, 2 \mathrm{H}), 1.66(\mathrm{~s}, 1.5 \mathrm{H})$, $1.54(\mathrm{~s}, 1.5 \mathrm{H}), 1.35(\mathrm{~m}, 10 \mathrm{H}) ;{ }^{13} \mathrm{C} \mathrm{NMR}\left(\mathrm{CDCl}_{3}, 100 \mathrm{MHz}\right) \delta 171.3,159.3,159.3,144.6$ $144.5,139.2,139.0,139.0,138.9,138.9,137.0,136.2,136.0,128.6,127.9,127.6,124.1$, $123.4,114.3,114.3,112.2,112.1,111.4,103.5,70.0,39.5,37.9,36.5,36.2,33.8,33.7$, $31.6,29.7,29.6,29.1,28.9,28.8,28.7,28.5,27.4,27.4,25.5,23.4,15.9$; IR (film) $v_{\max }$ 
$3299,3074,2927,2854,1658,1614,1604,1556,1454,1434,1155,908,696 \mathrm{~cm}^{-1}$; HRMS (TOF-ES+) found $488.3534\left(\mathrm{M}+\mathrm{H}^{+}\right)$, calcd 488.3529 for $\mathrm{C}_{33} \mathrm{H}_{46} \mathrm{NO}_{2}$.

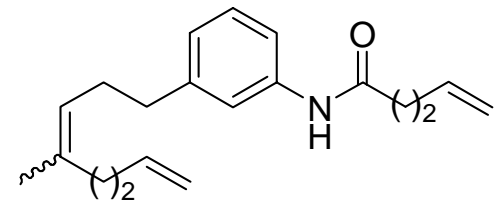

20: ${ }^{1} \mathrm{H}$ NMR $\left(\mathrm{CDCl}_{3}, 400 \mathrm{MHz}\right) \delta 7.36(\mathrm{~m}, 2 \mathrm{H}), 7.25(\mathrm{~m}, 2 \mathrm{H}), 6.95(\mathrm{~m}, 1 \mathrm{H}), 5.86(\mathrm{~m}$, 2H), $5.04(\mathrm{~m}, 5 \mathrm{H}), 2.62(\mathrm{~m}, 2 \mathrm{H}), 2.48(\mathrm{~m}, 4 \mathrm{H}), 2.30(\mathrm{~m}, 2 \mathrm{H}), 2.10(\mathrm{~m}, 4 \mathrm{H}), 1.70(\mathrm{~s}, 1.8 \mathrm{H})$, $1.57(\mathrm{~s}, 1.4 \mathrm{H}) ;{ }^{13} \mathrm{C} \mathrm{NMR}\left(\mathrm{CDCl}_{3}, 100 \mathrm{MHz}\right) \delta 170.9,143.8,143.8,139.1,139.0,138.2$, $138.2,137.3,135.8,135.8,129.2,129.2,125.0,124.9,124.9,124.2,120.3,120.2,117.7$, $117.6,116.3,114.8,114.7,39.4,37.3,36.7,36.5,32.7,32.6,31.7,30.2,30.1,29.9,23.7$, 16.4; IR (film) $v_{\max } 3299,3078,2923,2854,1660,1641,1612,1593,1552,1488,1440$, 1315, 993, 912, 786, $696 \mathrm{~cm}^{-1}$; HRMS (TOF-ES+) found $298.2178\left(\mathrm{M}+\mathrm{H}^{+}\right)$, calcd 298.2171 for $\mathrm{C}_{20} \mathrm{H}_{28} \mathrm{NO}$.

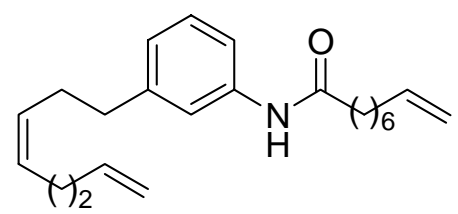

21: ${ }^{1} \mathrm{H}$ NMR $\left(\mathrm{CDCl}_{3}, 400 \mathrm{MHz}\right) \delta 7.41(\mathrm{~s}, 1 \mathrm{H}), 7.34(\mathrm{~d}, J=8.1 \mathrm{~Hz}, 1 \mathrm{H}), 7.24(\mathrm{dd}, J=8.1$, $7.4 \mathrm{~Hz}, 1 \mathrm{H}), 7.12(\mathrm{~s}, 1 \mathrm{H}), 6.96(\mathrm{~d}, J=7.4 \mathrm{~Hz}, 1 \mathrm{H}), 5.83(\mathrm{~m}, 2 \mathrm{H}), 5.41(\mathrm{~m}, 2 \mathrm{H}), 4.97(\mathrm{~m}$, 4H), $2.66(\mathrm{t}, J=7.7 \mathrm{~Hz}, 2 \mathrm{H}), 2.36(\mathrm{~m}, 4 \mathrm{H}), 2.05(\mathrm{~m}, 6 \mathrm{H}), 1.73(\mathrm{~m}, 2 \mathrm{H}), 1.42(\mathrm{~m}, 6 \mathrm{H}) ;{ }^{13} \mathrm{C}$ NMR $\left(\mathrm{CDCl}_{3}, 125 \mathrm{MHz}\right) \delta 171.2,143.1,139.0,138.4 .137 .9,129.8,129.0,128.9,124.4$, $119.8,117.2,114.6,114.3,37.8,35.9,33.7,29.1,29.0,28.8,28.7,26.7,25.5$; IR (film) 
$v_{\max } 3298,2925,2854,1660,1641,1612,1593,1552,1488,1440,995,910,786,698$

$\mathrm{cm}^{-1}$; HRMS (TOF-ES+) found $340.2645\left(\mathrm{M}+\mathrm{H}^{+}\right)$, calcd 340.2640 for $\mathrm{C}_{23} \mathrm{H}_{34} \mathrm{NO}$.<smiles>C=CC=CCCc1cccc(NC(=O)C=C)c1</smiles>

24: ${ }^{1} \mathrm{H}$ NMR $\left(\mathrm{CDCl}_{3}, 400 \mathrm{MHz}\right) \delta 7.41(\mathrm{~s}, 1 \mathrm{H}), 7.34(\mathrm{~d}, J=8.0 \mathrm{~Hz}, 1 \mathrm{H}), 7.24(\mathrm{dd}, J=8.0$, $7.4 \mathrm{~Hz}, 1 \mathrm{H}), 7.13(\mathrm{~s}, 1 \mathrm{H}), 6.96(\mathrm{~d}, J=7.4 \mathrm{~Hz}, 1 \mathrm{H}), 5.81(\mathrm{~m}, 2 \mathrm{H}), 5.39(\mathrm{~m}, 2 \mathrm{H}), 5.00(\mathrm{~m}$, 4H), $2.65(\mathrm{t}, J=7.8 \mathrm{~Hz}, 2 \mathrm{H}), 2.36(\mathrm{~m}, 4 \mathrm{H}), 2.04(\mathrm{~m}, 6 \mathrm{H}), 1.75(\mathrm{~m}, 2 \mathrm{H}), 1.37(\mathrm{~m}, 10 \mathrm{H})$; ${ }^{13} \mathrm{C}$ NMR $\left(\mathrm{CDCl}_{3}, 125 \mathrm{MHz}\right) \delta 171.7,143.6,139.5,139.4,138.3,131.0,129.2,129.0$, $124.8,120.2,117.6,114.7,114.7,38.2,36.4,34.1,34.1,29.5,29.5,29.5,29.2,29.1,28.9$, 27.5, 26.0; IR (film) $v_{\max } 3298,2927,2854,1660,1641,1612,1593,1552,1488,1440$, 993, 910, 786, $698 \mathrm{~cm}^{-1}$; HRMS (TOF-ES+) found $368.2954\left(\mathrm{M}+\mathrm{H}^{+}\right)$, calcd 368.2953 for $\mathrm{C}_{25} \mathrm{H}_{38} \mathrm{NO}$.

\section{Typical procedure for Ring-Closing Metathesis reaction}

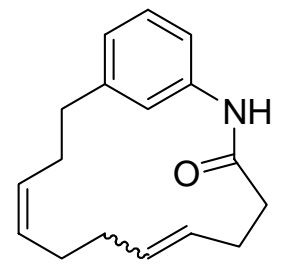

19 (5.6 mg, 0,02 mmol) was dissolved in $\mathrm{CH}_{2} \mathrm{Cl}_{2}(400 \mathrm{~mL})$ before argon was passed through the solution for 30 min to remove $\mathrm{O}_{2} .1^{\text {st }}$ generation Grubbs' catalyst $(1 \mathrm{mg})$ was added and the homogeneous mixture stirred for $12 \mathrm{~h}$ before an additional $1 \mathrm{mg}$ of Grubbs' catalyst was added. The solution was stirred for $12 \mathrm{~h}$ before passing air through 
the solution. After concentration, the residue was purified via chromatography $\left(\mathrm{SiO}_{2}\right.$, $30 \%$ EtOAc in hexanes) to afford $27(2.3 \mathrm{mg}, 45 \%$ yield $)$ as a white solid: ${ }^{1} \mathrm{H}$ NMR $\left(\mathrm{CDCl}_{3}, 400 \mathrm{MHz}\right) \delta 7.65(\mathrm{~m}, 1 \mathrm{H}), 7.22(\mathrm{~m}, 1 \mathrm{H}), 7.13(\mathrm{~m}, 1 \mathrm{H}), 6.98(\mathrm{~s}, 1 \mathrm{H}), 6.88(\mathrm{~m}, 1 \mathrm{H})$, $5.85(\mathrm{~m}, 0.73 \mathrm{H}), 5.63(\mathrm{~m}, 0.73 \mathrm{H}), 5.36(\mathrm{~m}, 2.54 \mathrm{H}), 2.76(\mathrm{~m}, 2 \mathrm{H}), 2.35(\mathrm{~m}, 9 \mathrm{H}), 2.07(\mathrm{~m}$, $0.71 \mathrm{H}), 1.78(\mathrm{~m}, 0.29 \mathrm{H}) ;{ }^{13} \mathrm{C} \mathrm{NMR}\left(\mathrm{CDCl}_{3}, 125 \mathrm{MHz}\right) \delta 170.9,142.6,137.8,133.9$, $132.4,130.0,129.7,128.8,124.9,118.4,117.1,36.0,32.7,32.1,29.5,27.7,26.1$; IR (film) $v_{\max } 3440,3271,2920,1658,1610,1593,1537,1479,1444,1274,1097,773,690$ $\mathrm{cm}^{-1}$; HRMS (TOF-ES+) found $256.1696\left(\mathrm{M}+\mathrm{H}^{+}\right)$, calcd 256.1701 for $\mathrm{C}_{17} \mathrm{H}_{22} \mathrm{NO}$.

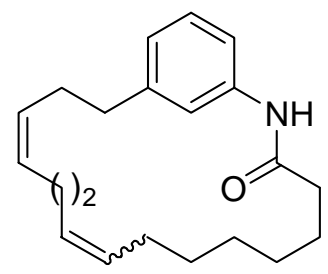

cis-29: ${ }^{1} \mathrm{H}$ NMR $\left(\mathrm{CDCl}_{3}, 400 \mathrm{MHz}\right) \delta 7.45(\mathrm{~m}, 1 \mathrm{H}), 7.24(\mathrm{~m}, 1 \mathrm{H}), 7.18(\mathrm{~m}, 1 \mathrm{H}), 7.10(\mathrm{~m}$, 1H), $6.85(\mathrm{~m}, 1 \mathrm{H}), 5.27(\mathrm{~m}, 4 \mathrm{H}), 2.66(\mathrm{t}, J=7.0 \mathrm{~Hz}, 2 \mathrm{H}), 2.36(\mathrm{~m}, 4 \mathrm{H}), 1.97(\mathrm{~m}, 3 \mathrm{H})$, $1.78(\mathrm{~m}, 3 \mathrm{H}), 1.53(\mathrm{~m}, 1 \mathrm{H}), 1.37(\mathrm{~m}, 7 \mathrm{H}) ;{ }^{13} \mathrm{C} \mathrm{NMR}\left(\mathrm{CDCl}_{3}, 125 \mathrm{MHz}\right) \delta 171.3,142.9$, 138.0, 130.8, 130.5, 129.7, 128.6, 128.2, 125.5, 119.4, 117.0, 37.5, 35.5, 32.3, 31.6, 29.1, 28.6, 28.2, 27.3, 27.2, 25.0; IR (film) $v_{\max } 3296,2923,2852,1658,1612,1593,1552$, 1487, 1442, 1303, 1261, 966, 784, $696 \mathrm{~cm}^{-1}$; HRMS (TOF-ES+) found 312.2353 $\left(\mathrm{M}+\mathrm{H}^{+}\right)$, calcd 312.2327 for $\mathrm{C}_{21} \mathrm{H}_{30} \mathrm{NO}$.

tran-29: ${ }^{1} \mathrm{H}$ NMR $\left(\mathrm{CDCl}_{3}, 400 \mathrm{MHz}\right) \delta 7.36(\mathrm{~m}, 1 \mathrm{H}), 7.23(\mathrm{~m}, 2 \mathrm{H}), 7.08(\mathrm{~m}, 1 \mathrm{H}), 6.93$ $(\mathrm{m}, 1 \mathrm{H}), 5.32(\mathrm{~m}, 4 \mathrm{H}), 2.70(\mathrm{t}, J=7.0 \mathrm{~Hz}, 2 \mathrm{H}), 2.37(\mathrm{~m}, 4 \mathrm{H}), 2.00(\mathrm{~m}, 2 \mathrm{H}), 1.89(\mathrm{~m}, 4 \mathrm{H})$, $1.77(\mathrm{~m}, 2 \mathrm{H}), 1.38(\mathrm{~m}, 6 \mathrm{H}) ;{ }^{13} \mathrm{C} \mathrm{NMR}\left(\mathrm{CDCl}_{3}, 125 \mathrm{MHz}\right) \delta 171.5,143.1,137.6,130.2$, $130.2,129.4,128.8,128.7,125.2,120.3,117.7,37.8,35.4,28.8,28.7,27.9,27.6,27.5$, 
27.3, 26.2, 25.3; IR (film) $v_{\max } 3292,2923,2852,1656,1612,1593,1552,1488,1442$, 1292, 1263, 784, 723, $696 \mathrm{~cm}^{-1}$; HRMS (TOF-ES+) found $312.2348\left(\mathrm{M}+\mathrm{H}^{+}\right)$, calcd 312.2327 for $\mathrm{C}_{21} \mathrm{H}_{30} \mathrm{NO}$.

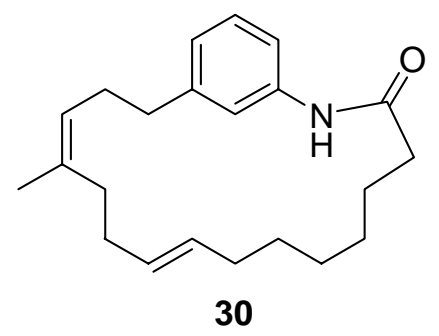

30: ${ }^{1} \mathrm{H}$ NMR $\left(\mathrm{CDCl}_{3}, 400 \mathrm{MHz}\right) \delta 7.52(\mathrm{~s}, 1 \mathrm{H}), 7.22(\mathrm{~m}, 2 \mathrm{H}), 7.06(\mathrm{~m}, 1 \mathrm{H}), 6.97(\mathrm{~m}, 1 \mathrm{H})$, $5.29(\mathrm{~m}, 2 \mathrm{H}), 5.09(\mathrm{t}, J=7.1 \mathrm{~Hz}, 1 \mathrm{H}), 2.67(\mathrm{~m}, 2 \mathrm{H}), 2.33(\mathrm{~m}, 4 \mathrm{H}), 2.01(\mathrm{~m}, 4 \mathrm{H}), 1.88(\mathrm{~m}$, 2H), $1.72(\mathrm{~m}, 2 \mathrm{H}), 1.36(\mathrm{~m}, 9 \mathrm{H}) ;{ }^{13} \mathrm{C} \mathrm{NMR}\left(\mathrm{CDCl}_{3}, 125 \mathrm{MHz}\right) \delta 171.2,143.1,137.3$, 136.6, 129.9, 129.6, 128.8, 124.6, 122.0, 121.0, 116.7, 39.9, 38.1, 35.6, 29.1, 28.1, 27.4, 27.2, 26.4, 25.5, 25.3, 16.2; IR (film) $v_{\max } 3296,2923,2854,1656,1612,1593,1552$, 1488, 1440, 1294, 1263, 786, $698 \mathrm{~cm}^{-1}$; HRMS (TOF-ES+) found $326.2508\left(\mathrm{M}+\mathrm{H}^{+}\right)$, calcd 326.2484 for $\mathrm{C}_{22} \mathrm{H}_{32} \mathrm{NO}$.

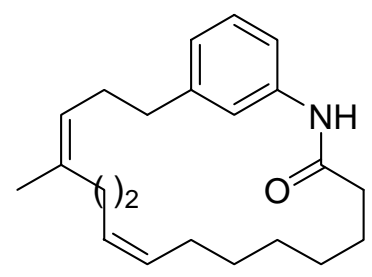

31

31: ${ }^{1} \mathrm{H}$ NMR $\left(\mathrm{CDCl}_{3}, 400 \mathrm{MHz}\right) \delta 7.31(\mathrm{~s}, 1 \mathrm{H}), 7.22(\mathrm{~m}, 2 \mathrm{H}), 7.06(\mathrm{~m}, 1 \mathrm{H}), 6.94(\mathrm{~m}, 1 \mathrm{H})$, $5.29(\mathrm{~m}, 2 \mathrm{H}), 5.17(\mathrm{~m}, 1 \mathrm{H}), 2.65(\mathrm{t}, J=7.8 \mathrm{~Hz}, 2 \mathrm{H}), 2.30(\mathrm{~m}, 4 \mathrm{H}), 2.02(\mathrm{~m}, 6 \mathrm{H}), 1.78(\mathrm{~m}$, $2 \mathrm{H}), 1.68(\mathrm{~s}, 3 \mathrm{H}), 1.40(\mathrm{~m}, 6 \mathrm{H}) ;{ }^{13} \mathrm{C} \mathrm{NMR}\left(\mathrm{CDCl}_{3}, 125 \mathrm{MHz}\right) \delta 171.7,143.5,137.5$, $135.9,130.1,129.6,128.8,125.3,124.5,120.8,118.4,37.7,36.0,32.4,30.2,29.3,28.4$, 
27.9, 26.4, 25.9, 25.4, 23.5; IR (film) $v_{\max } 3259,2925,2852,1652,1610,1593,1539$, 1481, 1440, 788, $698 \mathrm{~cm}^{-1}$; HRMS (TOF-ES+) found 348,2331 $\left(\mathrm{M}+\mathrm{Na}^{+}\right)$, calcd 348.2303 for $\mathrm{C}_{22} \mathrm{H}_{31} \mathrm{NONa}$.

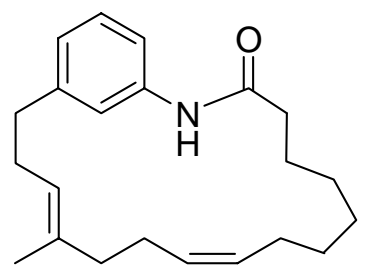

32

32: ${ }^{1} \mathrm{H}$ NMR $\left(\mathrm{CDCl}_{3}, 400 \mathrm{MHz}\right) \delta 7.40(\mathrm{~s}, 1 \mathrm{H}), 7.21(\mathrm{~m}, 1 \mathrm{H}), 7.11(\mathrm{~m}, 1 \mathrm{H}), 6.98(\mathrm{~m}, 1 \mathrm{H})$, $6.84(\mathrm{~m}, 1 \mathrm{H}), 5.28(\mathrm{~m}, 2 \mathrm{H}), 5.13(\mathrm{~m}, 1 \mathrm{H}), 2.62(\mathrm{t}, J=7.3 \mathrm{~Hz}, 2 \mathrm{H}), 2.32(\mathrm{~m}, 4 \mathrm{H}), 1.97(\mathrm{~m}$, 4H), $1.85(\mathrm{~m}, 3 \mathrm{H}), 1.62(\mathrm{~m}, 4 \mathrm{H}), 1.37(\mathrm{~m}, 6 \mathrm{H}) ;{ }^{13} \mathrm{C} \mathrm{NMR}\left(\mathrm{CDCl}_{3}, 125 \mathrm{MHz}\right) \delta$ 171.3, 143.4, 137.9, 136.4, 130.7, 129.4, 128.6, 125.4, 123.9, 119.7, 117.4, 37.4, 36.1, 32.0, 31.7, 30.9, 30.3, 28.8, 28.0, 27.3, 24.9, 23.4; IR (film) $v_{\max } 3294,2923,2852,1658,1612$, 1593, 1552, 1487, 1442, 966, 784, $696 \mathrm{~cm}^{-1}$; HRMS (TOF-ES+) found 326.2507 $\left(\mathrm{M}+\mathrm{H}^{+}\right)$, calcd 326.2484 for $\mathrm{C}_{22} \mathrm{H}_{32} \mathrm{NO}$.

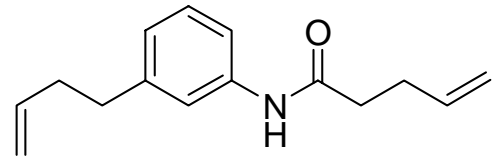

33: ${ }^{1} \mathrm{H}$ NMR $\left(\mathrm{CDCl}_{3}, 400 \mathrm{MHz}\right) \delta 7.42(\mathrm{~s}, 1 \mathrm{H}), 7.31(\mathrm{~d}, J=8.2 \mathrm{~Hz}, 1 \mathrm{H}), 7.24(\mathrm{~m}, 2 \mathrm{H})$, $6.95(\mathrm{~d}, J=7.4 \mathrm{~Hz}, 1 \mathrm{H}), 5.88(\mathrm{~m}, 2 \mathrm{H}), 5.07(\mathrm{~m}, 4 \mathrm{H}), 2.71(\mathrm{t}, J=7.8 \mathrm{~Hz}, 2 \mathrm{H}), 2.49(\mathrm{~m}$, 4H), $2.38(\mathrm{~m}, 2 \mathrm{H}) ;{ }^{13} \mathrm{C} \mathrm{NMR}\left(\mathrm{CDCl}_{3}, 125 \mathrm{MHz}\right) \delta 170.9,143.4,138.3,138.2,137.3$, 129.3, 124.9, 120.2, 117.7, 116.4, 115.4, 37.3, 35.8, 35.7, 29.8; IR (film) $v_{\max } 3299,3078$, $2925,2854,1660,1641,1612,1593,1552,1488,1440,1309,1261,995,912,786,698$ $\mathrm{cm}^{-1}$; HRMS (TOF-ES+) found $230.1548\left(\mathrm{M}+\mathrm{H}^{+}\right)$, calcd 230.1545 for $\mathrm{C}_{15} \mathrm{H}_{20} \mathrm{NO}$. 


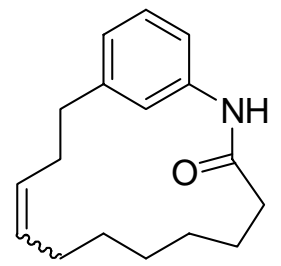

34: ${ }^{1} \mathrm{H}$ NMR $\left(\mathrm{CDCl}_{3}, 400 \mathrm{MHz}\right) \delta 7.48(\mathrm{~m}, 0.49 \mathrm{H}), 7.30(\mathrm{~m}, 0.51 \mathrm{H}), 7.19(\mathrm{~m}, 2 \mathrm{H}), 6.92$ (m, 2H), $5.25(\mathrm{~m}, 2 \mathrm{H}), 2.73(\mathrm{~s}, 2 \mathrm{H}), 2.25(\mathrm{~m}, 4 \mathrm{H}), 1.94(\mathrm{~m}, 3 \mathrm{H}), 1.70(\mathrm{~m}, 2 \mathrm{H}), 1.26(\mathrm{~m}$, 5H), $0.96(\mathrm{~m}, 1 \mathrm{H}) ;{ }^{13} \mathrm{C} \mathrm{NMR}\left(\mathrm{CDCl}_{3}, 125 \mathrm{MHz}\right) \delta 176.0,172.2,143.5,142.9,136.4$, $132.8,132.3,130.3,129.2,128.7,128.2,128.1,125.3,124.8,123.2,118.5,38.9,34.5$, $34.3,34.1,33.4,33.0,31.8,30.4,30.1,29.6,28.2,26.5,26.0,25.6,23.6$; IR (film) $v_{\max }$ $3440,3247,3143,3070,2923,2852,1654,1593,1558,1548,1481,1446,1431,1363$, 1282, 1195, 1103, 962, 879, 794, 734, $702 \mathrm{~cm}^{-1}$; HRMS (TOF-ES+) found 258.1879 $\left(\mathrm{M}+\mathrm{H}^{+}\right)$, calcd 258.1858 for $\mathrm{C}_{17} \mathrm{H}_{24} \mathrm{NO}$.

${ }^{a}$ Sinisterra, J. V.; Marinas, J. M.; Riquelme, F.; Arias, M. S. Tetrahedron 1988, 44, 14311440.

${ }^{\mathrm{b}}$ Fuchs, R.; Caputo, J. A. J. Org. Chem. 1966, 31, 1524-1526.

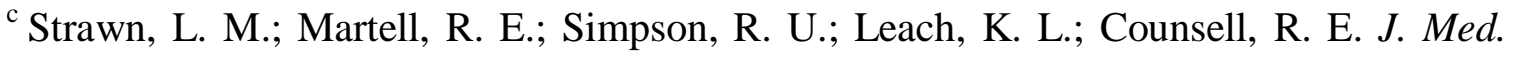
Chem. 1989, 32, 2104-2110. 